\title{
Dramatic Increase of Multiresistant Microorganisms
}

\author{
Guggenbichler $\mathbf{S}^{1}$ and Guggenbichler JP ${ }^{2 *}$ \\ ${ }^{1}$ Division for Vascular Surgery and Endovascular Surgery of the University Clinic for Cardiac Surgery, Vascular Surgery and \\ Endovascular Surgery, Austria
}

${ }^{2}$ AmiSTec GmbH and co KG, Kössen, Austria

*Corresponding author: J Peter Guggenbichler, AmiSTec GmbH and co KG, Kössen, route 23, Austria

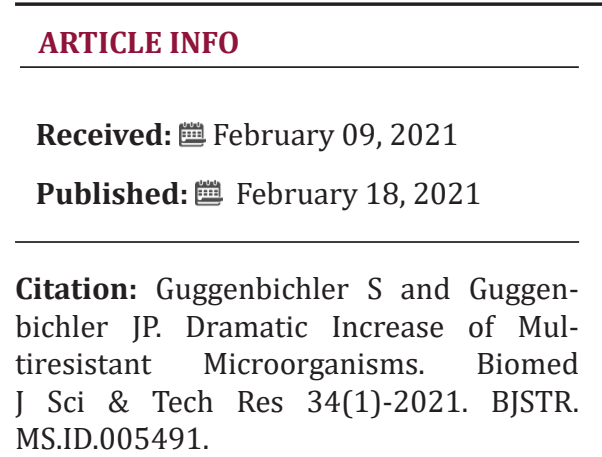

Abbreviations: FDA: Food and Drug Administration; QAC: Quaternary Ammonium Base Disinfectant; AMPs: Antimicrobial Peptides; QACs: Quaternary Ammonium Compounds; CFU: ColonyForming Units

\begin{abstract}
For people in the 21st century, it is hard to imagine the world before antibiotics. At the beginning of the 20th century, as many as nine women out of every 1,000 who gave birth died, 40 percent from sepsis. In some cities as many as 30 percent of children died before their first birthday. One of every nine people who developed a serious skin infection died, even from something as simple as a scrape or an insect bite. Pneumonia killed 30 percent of those who contracted it; bacterial meningitis was almost universally fatal. Ear infections caused deafness; sore throats were not infrequently followed by rheumatic fever and heart failure. Surgical procedures were associated with high morbidity and mortality due to infection. This picture changed dramatically with three major developments: improvements in public health, vaccines, and antibiotics. Over the course of the $20^{\text {th }}$ century, deaths from infectious diseases declined markedly and contributed to a substantial increase in life expectancy.
\end{abstract}

Antibiotics, in particular, have saved millions of lives. But the world is now at dire risk of losing this progress. Bacteria and other microbes evolve in response to their environment and inevitably develop mechanisms to resist being killed by antibiotics but also disinfectants $[1,2]$. For many decades, the problem was manageable as the growth of resistance was slow and the pharmaceutical industry continued to create new antibiotics. Over the past decade, however, this brewing problem has become a crisis. The evolution of antibiotic resistance is now occurring at an alarming rate and is outpacing the development of new countermeasures capable of thwarting infections in humans [3]. This situation threatens patient care, economic growth, public health, agriculture, economic security, and national security. The UN Interagency Coordination Group on Antimicrobial Resistance demands therefore immediate, coordinate and ambitious measures to combat this problem [4].

\section{The Recommendations are:}

a) Investigations to improve our surveillance of the rise of antibiotic-resistant bacteria is required to enable an effective response, stop outbreaks, and limit the spread of antibioticresistant organisms to implement appropriate infection control.

b) The next goal is to increase the longevity of current antibiotics, by improving the appropriate use of existing antibiotics, preventing the spread of antibiotic-resistant bacteria and scaling up proven interventions to decrease the rate at which microbes develop resistance to current antibiotics.

c) Increasing the rate at which new antibiotics, as well as other interventions, e.g., alternative antimicrobial substances and modes of application are developed.

\section{Human Health Care}

Use and Overuse of Antibiotics: Antibiotics are among the most commonly prescribed drugs used in human medicine. CDC estimates, however, that up to 50 percent of all the antibiotics prescribed for patients are not needed or are not optimally prescribed [5]. Reasons for antibiotic overuse in health care include lack of rapid diagnostic tests and pressure from the patient (or the patient's family) due to insufficient understanding of antibiotic use. This misuse and overuse of antibiotics in human medicine, both in the United States and internationally, is a major contributor to rising antibiotic resistance in individual patients [6]. The impact however on the development of multi resistant microorganisms in 
Intensive care units however is limited and greatly overestimated. Fact is, that bacterial superinfection ensues in $25-40 \%$ of infants and children as a consequence of a viral respiratory tract infection by clogging the paranasal opening, the Eustachian tube and impairing mucociliary clearance.

Stagnant secretions in the paranasal sinus and the middle ear cavity are contaminated with bacterial microorganisms and result in serious infections - early administration of antibiotics it thought to prevent this. However, this is less effective than anticipated and induces simultaneously the emergence of resistant microorganisms. It is feasable to prevent bacterial superinfections by antiinflammatory properties and improvement of the mucociliary clearance of various herbal extracts e.g., triterpenes and 1.8. Cineol, contained in a number of herbal extracts e.g., thyme, gentian, sorrel, primulae, 1.8. cineol [7].

a) These extracts contain substances which decrease the formation of proinflammatory cytokines by inhibition of the arachidonic acid metabolism-the precursors of cytokines. By those means they exhibit strong anti-inflammatory properties which prevent the emergence of submucous edema responsible for bacterial superinfections of paranasal sinus and middle ear cavity. Simultaneously these herbal remedies improve the mucociliary clearance by increasing ciliary motility, harmonization of the viscosity of nasal and tracheal secretion. Clinical trials CPMP/ICH/135/95 show excellent efficacy with complete lack of side effects.

\section{Animal Agriculture}

Medically important antibiotics are also extensively used in animal agriculture not only to treat sick animals, but also to promote animal growth and to prevent infections. All of these uses promote the development of antibiotic resistance among bacteria in animals, and these resistant strains do, at least in some cases, spread to humans. Antibiotics administered to piglets prevent diarrhea and death and also result in improved gain of weight. Usually non absorbable antibiotics e.g., colistin are administered. These antibiotics select resistant microorganisms in the faecal flora which are excreted by slurry. This is distributed as fertilizers in the environment contaminating vegetables and fruits. The extent to which antibiotic resistance in animal agriculture contributes to human infections is a matter of controversy, the risks to human health posed by the agricultural use of antibiotics are however, appropriately, a matter of serious concern. There is a highly efficient innovative technology which prevents adherence and colonization of mucous membranes in the gut a necessary requirement for pathogenicity of pathogenic microorganisms.

Gram negative bacteria, mainly E. coli adhere on gal 1-4 gal structures on epithelial cells with fimbriae. Pectin is composed of several gal 1-4 galactose molecules. These di- or tri terpenes i.e., acid galacturonides obtained by cooking of pectines function as receptor analogues and block as receptor analogue carbohydrates the adherence of bacterial microorganisms on epithelial cells; the microorganisms are eliminated in the faeces without induction of resistance [8]. Figure 1 shows the prevention of diarrhea in piglets by comparing acid galacturonides versus an antibiotic (tylosin phosphate). The galacturonide turns out to be more effective in prevention of diarrhea than the antibiotic just as well as prevention of death due to adnexitis in a laying hen population. Figure 2 Herbal extracts are effective, not toxic and highly cost effective.

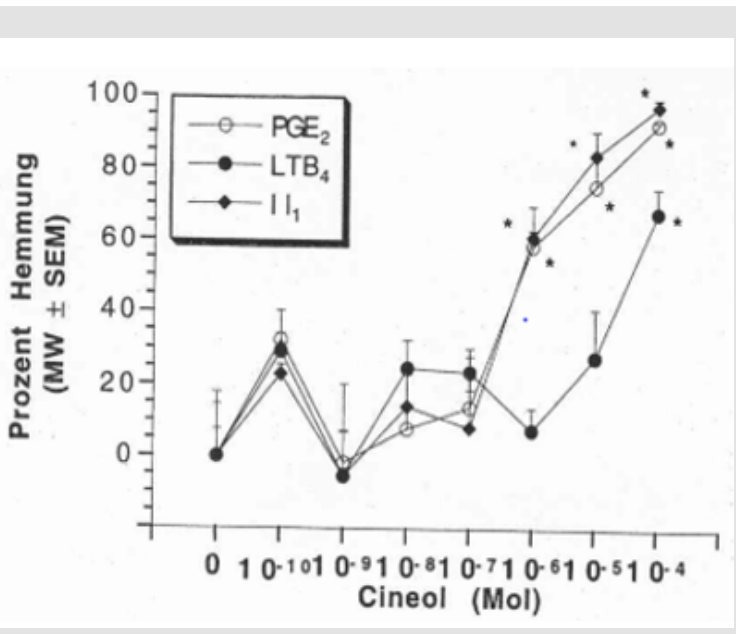

Figure 1.

Tab.1 Durchfallhäufigkeit in den Gruppen

\begin{tabular}{|c|c|c|c|}
\hline Gruppe & Mit Durchial & Ohne Durch'́ll & Gesamt \\
\hline Karotte & $10 \quad 14,7 \%$ & $58 \quad 85,3 \%$ & $68 \quad 100 \%$ \\
\hline Kontrolle & $31 \quad 50,0 \%$ & $31 \quad 50,0 \%$ & $62 \quad 100 \%$ \\
\hline LF nomingerse & $17 \quad 24,6 \%$ & $52 \quad 75,4 \%$ & $69 \quad 100 \%$ \\
\hline Gesant & $58 \quad 29,1 \%$ & $141 \quad 70,9 \%$ & $199 \quad 100 \%$ \\
\hline
\end{tabular}

Figure 2: Prophylaxis of diarrhea in newborn piglets by acid galacturonides in comparison with an antibiotic (Tylosine Phosphate) and a negative control.

\section{Increasing the Longevity of Current Antibiotics}

by improving the appropriate use of existing antibiotics and thereby preventing the spread of antibiotic-resistant bacteria. Certain antibiotics in use favour the emergence of resistant microorganisms in the fecal flora. Broad spectrum antibiotics with incomplete bioavailability e.g., cefixim with a bioavailability of $75 \%$ selects cephalosporin resistant microorganisms in the fecal flora which can contribute to bacterial superinfections e.g., urinary tract infections with multi resistant gram negative pathogens. Absorption can be facilitated by modification of the original drug to optimize absorption, distribution, metabolism, and excretion 
of these drugs. Formation of an ester is an example of a prodrug methodology for modification of a functional group of the active drug to improve lipophilicity for passive membrane transport or to increase aqueous solubility.

Such broad-spectrum antibiotic shows an excellent bioavailability but if eliminated by the biliary route - an accumulation of unabsorbed antimicrobial active substance in the large intestine is observed again with selection of multi resistant microorganisms. For prodrugs renal elimination is therefore required [7]. Another problem is an excessive long half-life of an antibiotic. The usually observed half-life of a macrolide antibiotic is 60 minutes. Azitromycin has a halflife to 3 days which results in subinhibitory concentrations in the oral cavity responsible for the induction of macrolide resistant microorganisms. In addition, the initial poor absorption does not result in rapidly bactericidal concentration at the site of an infection [10]. It is therefore necessary for the approval of new antibiotics to take also pharmacokinetic and pharmacodynamics properties of antimicrobial agents into serious consideration (Figures 3-5).

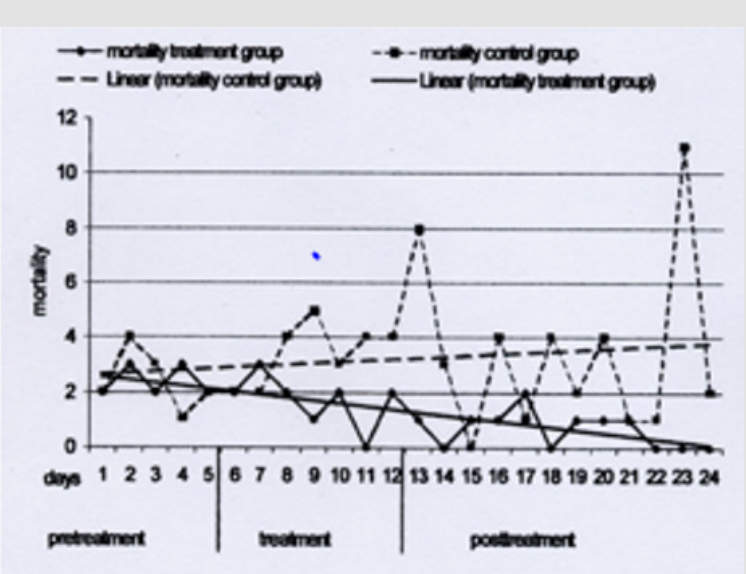

Figure 3: Treatment of an outbrake of adnexitis in a laying hen population with herbal extracts.

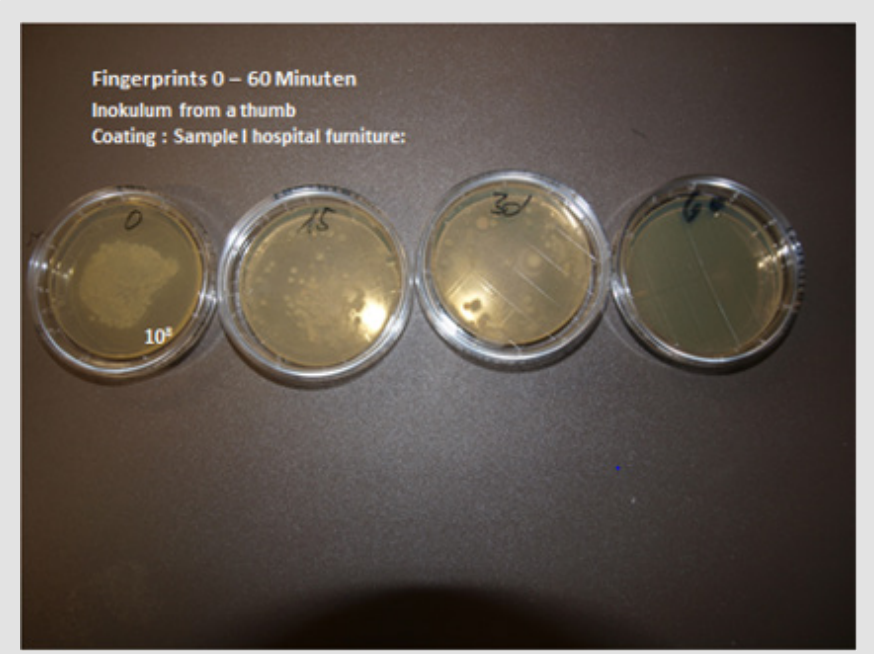

Figure 4 .

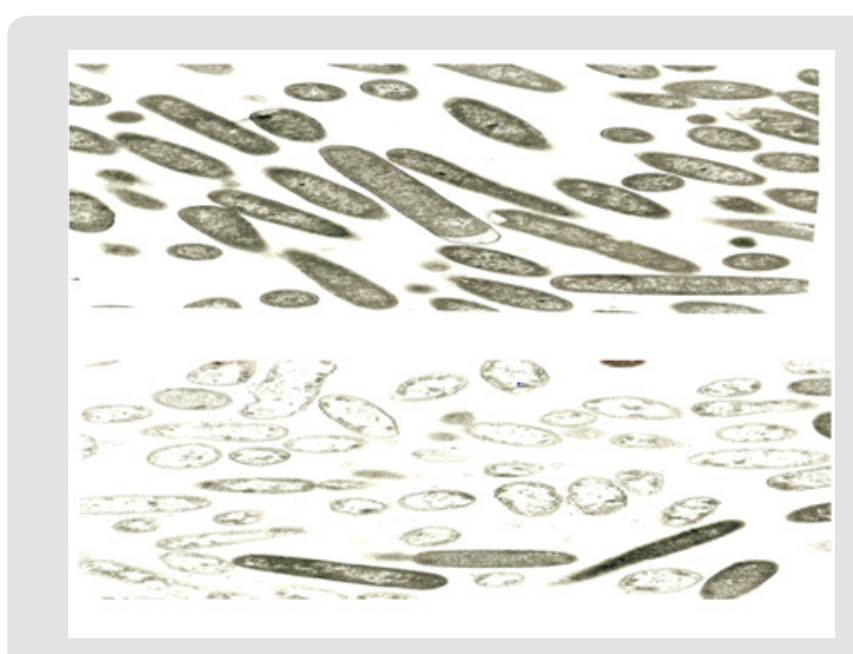

Figure 5.

\section{Drug Development}

The world lacks a robust pipeline of new antibiotics to replace those being steadily lost to antibiotic resistance. The number of new systemic antibacterial agents approved by the Food and Drug Administration (FDA) each year has decreased steadily over the past three decades. The drug development pipeline is failing for a variety of reasons-including scientific challenges in discovering and developing new drugs; practical challenges in conducting the clinical trials needed for new-drug approval; and low economic returns that have made the development of new antibiotics an unattractive investment. As a result, large pharmaceutical companies have decreased or eliminated their investments in antibiotic drug development. There is an important observation which makes the development of new antimicrobial substances also less attractive. It has been observed that microorganisms develop resistance to new antimicrobial substances within a period of a few months. A law by nature indicates that every antimicrobial substance which has to be incorporated into the metabolism of microorganisms induces resistance. Alternative modes of antimicrobial activity have to be designed which are not connected with induction of resistance.

\section{Investigation of the Origin of Resistant Microorganisms}

10 years ago, a multi resistant microorganism has been isolated on a surface which has been just disinfected with a Quaternary Ammonium Base Disinfectant (QAC). Initially this was a surprise but initiated intensive investigative work. QAC resistance is widespread among a diverse range of microorganisms and is facilitated by several mechanisms such as modifications in the membrane composition, expression of stress response and repair systems, or expression of efflux pump genes which results in a widespread resistance against virtually all antibiotics. Development of resistance in both pathogenic and nonpathogenic bacteria has been related to application in human medicine and the food industry. The induction of resistance to disinfectants and cross resistance with 
antibiotics has been confirmed by the Norwegian food and fishery administration which prohibited the use of quaternary ammonium compound disinfectants in the fish industry [11].

Review of the international literature (pubmed) provides more than 8000 articles which describe the resistance of microorganisms against disinfectants and 800 papers report the cross resistance with antibiotics. Disinfectant resistance has the potential to change our way of life from threatening our medical health systems to compromising food security. Resistance to antimicrobial agents occurs through either intrinsic or acquired resistance mechanisms. Acquired resistance occurs through the efficient transfer of mobile genetic elements, which can carry single, or multiple resistance determinants. Drug resistance genes may form part of integrons, transposons and insertions sequences which are capable of intracellular transfer onto plasmids or gene cassettes.

Thereafter, resistance plasmids and gene cassettes mobilize by self-transmission between bacteria, increasing the prevalence of drug resistance determinants in a bacterial population. New methods, in addition or as an alternative to appropriate use of disinfectants and antibiotics, are required to reduce microbial associated infections and to reverse the increase in antimicrobial resistance. A promising alternative for the disinfection of surfaces are self-sanitizing surfaces which eradicate microorganisms fast from a surface and prevent the distribution of resistant microorganisms within the hospital by the personnel to patients. Bactericidal coatings are interesting in healthcare because of the capability of these coatings to kill pathogens upon contact. Many different chemical strategies and technologies for antimicrobial coatings are described in the literature with variable success.

It is well known that microorganisms in a biofilm cannot be eradicated by antibiotics nor disinfectants [12]. The initial investigative work excluded the theory, that antimicrobial substances are prevented from diffusion into the biofilm. Further investigations disclosed that microorganisms in a biofilm are hibernating and don't take up anything from the outside. However, they can be eradicated by a technology which affects the pathogens from the outside. The requirements of self-sanitizing surfaces for application in hospitals, public transportation, the food industry is extraordinarily high.

a) Intensive and broad antimicrobial activity, against Grampositive, Gram-negative microorganisms, irrespective of their antibiotic susceptibility, fungi, legionella, moulds, virus documented by the RODAC plate method.

b) Fast eradication of microorganisms i.e., minimum $5 \log 10$ reduction within 1 hour

c) Activity against a high inoculum size of $109 \mathrm{CFU}$ on an area of $3 \mathrm{~cm}^{2}$

d) No induction of resistance e) Nontoxic, skin and soft tissue compatibility, no allergenicity, sbD (safe by design)

f) Long lasting/permanent antimicrobial activity water-, acid-, alkaline-, alcohol insoluble, UV Light stabile

g) Cleanable with detergents

h) Uncomplicated technical processability, heat stabile up to $400^{\circ} \mathrm{C}$, non-corrosive

i) Physical stability, activity irrespective of sweat, grease, blood, pus.

j) Not flammable, smoke reduction

k) BP authorisation by the European commission on biocidal products.

l) Favourable cost/benefit analysis

Self-sanitizing surfaces are mostly focused on solid air interfaces in health-care units, such as tables, doorhandles, computer keyboards, textiles, although solid liquid surfaces are also of great concern in hospitals such as taps, showers and drains, where biofilms appear frequently. Last not least certain technologies for a self-sanitizing surface can also be used for implantable biomaterials.

Antibacterial Coatings May Contain Active Eluting Agents (e.g., ions or nanoparticles of silver, copper, zinc, or antibiotics, chloride, iodine, quaternary ammonium polymers or antimicrobial peptides, or light-activated molecules (e.g., $\mathrm{TiO}_{2}$ ), immobilized molecules that become active upon contact [13].

a) Active Eluting Agents Show Several Disadvantages: The active substances must be eluted from of the surface i.e. the polymer or the coating. This means that their activity is limited to a short period of time. For antibiotics this means a duration of activity of less than 3 days, for silver 3 weeks [14]. Copper cannot embedded into polymers to achieve a sufficient antimicrobial activity; in addition, the activity of copper is too low to be considered as a valuable compound [15]. Quaternary ammonium compounds must be excluded from considerations as these products may even enhance the growth of microorganisms on surfaces and induce cross resistance with antibiotics by induction of efflux pumps. Various drug eluting substances e.g., Polybiguanides, halogenated phenols, and polyethyleneimines have been investigated but showed toxic side effects with human tissues as they have to be eluted from the polymer of the surface [16-23].

\section{Chemical Modifications to Achieve Functional Antimicrobial Coatings}

In addition to chemical modifications, the topography of a surface can by itself significantly affect its hygienic status, either in a beneficial manner (reducing microbial retention) or otherwise (increasing retention). As such, modifications of surfaces to 
enhance antimicrobial properties should always consider the effect of surface wear on subsequent fouling and cleanability. Therefore, efforts should be undertaken to characterize typical wear, assess interactions with the most likely microorganisms in that environment, and define the most appropriate and least damaging cleaning and sanitizer regimes. Antimicrobial agents adjacent to a surface showed the risk of abrasion with cleaning The agents have not been consistently insoluble in water-, alcohol-, detergents, acid, alkaline, in addition UV light stability has not been confirmed. which is not guaranteed by several of the abovementioned compounds Strategies to achieve antimicrobial coatings can be classified according to their functional principle as: (i) antiadhesive, (ii) contact active, and (iii) biocide release.

Whereas the first two principles may be considered as SbD, biocide release incorporates the release of a toxic substance and can therefore be considered as toxic by design. Sometimes two functional principles are combined to achieve synergistic effects, e.g. by embedding biocidal substances into anti-adhesive surfaces. Today, the majority of chemical modifications includes hydrogels or poly (ethylene glycol) (PEG) to repel approaching microbes, metals (in particular, silver and copper), Antimicrobial Peptides (AMPs), Quaternary Ammonium Compounds (QACs), and nanoparticles [13e18]. Beyond those established approaches, state-of-the-art or potentially new strategies towards antimicrobial coatings were identified. For many of these antimicrobial strategies, the mechanism of antimicrobial activity is still under investigation and there is not enough information available on whether antimicrobial activity happens directly at the surface or whether small amounts of active compounds are released into the test media where they will exert their antimicrobial activity, or last whether both mechanisms are acting in parallel. Besides engagement with known antimicrobial agents' emphasis should have been exerted in evaluating

a) Documentation of the mechanism of action of presently available compounds

b) Investigation of new principles with new and promising antimicrobial activity.

\section{Anti-Adhesive Surfaces}

Anti-adhesive surfaces could reduce the adhesion force between bacteria and a solid surface to enable the easy removal of bacteria before a biofilm layer is formed on the surface [24]. Such surfaces may suppress HCAI by blocking transmission paths involving surfaces, but they will not reduce the number of germs on the contacting media by killing them. Attachment of bacteria or cells starts with an initial adsorption of proteins on to the material surface [20]. Strategies to prevent protein attachment include superhydrophobic surfaces, often augmented by a hierarchical nanostructure as well as zwitterionic polymers $[25,26]$. The most important requirement of a "self-sanitizing" surface is the ability of the surface to actively eradicate pathogens within a reasonable short period of time. It has to be emphasized that microorganisms are deposited by the hand of the personnel with considerable force. For prevention of recontamination of a second person, rapid eradication of microorganisms is mandatory (i.e., less than 1 hour or shorter).

Reduction of adherence, blockage of proliferation and biofilm formation although also important - are in no way sufficient as "self-sanitizing" surfaces for clinical use to prevent recontamination of persons. Contamination of a surface (melamine resin with $2 \%$ Zinc Molybdate) has been performed with thumb prints. The initial number of CFU on the surface is 108 CFU (lawn) on an area of $2 \mathrm{~cm}^{2}$. There is a substantial reduction of microorganisms after 15 and 30 minutes. At 60 minutes no germs are isolated on the surface. This has been investigated by the push plate (RODAC plate) method. Superhydrophobic surfaces are characterized by a water contact angle $>150$ " and they are inspired by the lotus leaf in nature [25]. It was further revealed that the lotus leaf has a hierarchical micro/nanostructure [26]. Reducing bacterial adhesion via superhydrophobicity is a relatively new topic and has yet to be studied thoroughly and systematically [27].

Analysis of superhydrophobic siloxane and fluor siloxane surfaces showed also minimal protein adsorption, both before and after protein adsorption trials [28]. Nanostructures are important, since effective air entrapment in the three-dimensional nanomorphology (nanopillars) renders them superhydrophobic and slippery. On inherently nanostructured hydrophilic aluminum, adhesion forces of bacteria were reduced by a factor of 4 down to $2 \mathrm{e} 4 \mathrm{nN}$ compared to the electropolished flat surface, resulting in an $88 \%$ reduction of Colony-Forming Units (CFU) for Staphylococcus aureus. This effect was even more pronounced after applying a hydrophobic Teflon coating, yielding a 99.9\% reduction under flow conditions. All these hyperhydrophobic properties require the nanotechnology with the problems of approval by the biocidal regulation authorities. Easier cleaning is not the answer - the surface must eradicate contaminating microorganisms fast. A potential and promising weapon against bacterial growth and possibly the development of multidrug-resistant bacteria has been found in antimicrobial (nano)coatings (AMC)

\section{Nanostructured Surfaces}

One proposed technology is the use of nanocoating's: Antimicrobial technologies using nanomaterials e.g. chitosan, cellulose etc. have to be applied into nanorods or nanosats which can however not stabil anchored on the surface. Nanostructured surfaces were also prepared using electro spun polystyrene nanofibers. When oxygen plasma-treated, a super hydrophilic surface was generated, which exhibited limited Escherichia coli attachment due to negative zeta potential of e $40 \mathrm{mV}$. After fluorination, a superhydrophobic surface was obtained, which exhibited self-cleaning ability against bacteria, where the initially adhered bacteria were effectively removed with subsequent 
washing [26]. Anti-adhesion and killing was achieved by combining an upper superhydrophobic surface layer (silane coated poly (acrylic acid)) with limited bacterial adhesion and self-cleaning properties with a hydrophilic bottom layer (poly(ethyleneimine)e Agp complex) which could deliver bactericidal silver ions [27].

Addition of silver has been described a technology for antimicrobial surfaces. One technology describes that free silver ions are antimicrobials active - where silver has to be released from the surface and incorporated into the bacterial metabolism. A strong disadvantage: a limited activity of silver of a few days has been described! Silver submicron particles can be incorporated into polymers and coatings, a hydrophilizing agent must also be added for an enhanced activity! Silver in form of nanoparticles function in a different way: Some studies have reported that nano-silver causes oxidative damage, leading to the production of reactive oxygen species (ROS) as well as free-radicals, and it has been suggested that the production of ROS is one of the primary mechanisms of nanoparticle toxicity. Hence, it was suggested that nano-silver affects bacterial membrane permeability by attaching to the cell membrane surface and modifying the cell potential.

Observation of large numbers of nanoparticles inside bacteria suggests that this is important to the antibacterial mechanism. An interesting anti-adhesive and killing approach is found in nature. The nano-patterned cicada wing surface uses an adsorption and stretching mechanism with eventual rupture. As the bacterial cells adsorb on to the nano pillared structures present on the wing surfaces, the bacterial cell membrane stretches in the regions suspended between the pillars. If the degree of stretching is sufficient, cell rupture will occur [28]. The cicadia wings technology as well as the gecko foot technology which relies on the same properties is not physically stabile the cicadia wings surface can be easily destroyed by mechanical wiping/cleaning, rubbing.

Zwitterionic polymer brushes may also delay or even prevent microbial attachment to a surface, since the hydration layer surrounding the ionic surface prevents non-specific protein adsorption [21]. Using barnacle cement, a biological adhesive from barnacles, and 'click' chemistry, poly(2-(methacryloyloxy) ethyl trimethylammonium chloride) polymer brushes were successfully attached to stainless steel and antimicrobial properties were demonstrated [27]. Zwitterionic polymer brushes cannot inactivate bacterial cells. Therefore, synergistic anti-adhesion and bacterial inactivation was achieved by grafting zwitterionic poly (sulfobetaine methacrylate) brushes with embedded biocidal silver nanoparticles [28]. Addition of silver is possible b u t only silver ions are active - in other words silver has to be released from the hydrophilic bottom layer and incorporated into the bacterial metabolism (mode of action well known) Limited activity to probably a few hours! The importance of anti-adhesive properties for biofilm formation was also demonstrated by measuring the adhesive forces on brush-coated silicone rubber and uncoated silicon rubber. On the brush-coated rubber, adhesion was so weak that the bacteria were no longer able to sense the surface and therefore remained in their planktonic state, susceptible to antibiotics rather than forming a protected biofilm.

\section{Contact-Active Surfaces}

Contact-active surfaces exhibit antimicrobial activity without releasing biocidal substances. Several mechanisms are believed to take place in contact-active surfaces. These are: (i) a so-called spacer effect, where the biocidal group is attached to the surface through a polymer chain, allowing the biocide to reach the cytoplasmic membrane of the bacteria and to perforate them; (ii) alternatively, positively charged QACs, e.g. 3-aminopropyl trimethoxysilane grafted to cellulose nanofibres, can detach phospholipids from the cell membrane and thereby kill the bacteria. This approach is also referred to as biomimetic with respect to the activity of chitosan a polysaccharide derived from exoskeleton of crustaceans or cell walls of fungi. Hydrophobic parts of a surface can act similarly to QACs by deforming the membrane through adhesion. The activity of the spacer effect is obliterated by grease, proteins, sweat, pus and blood. The activity of chitosan has been investigated and a poor antimicrobial activity has been found. Regarding to Nanotechnology and QACs see comment above.

Polymer brushes have been widely used in preparing contactactive antimicrobial surfaces without biocidal release. The rationale behind polymer brushes is the observation that antimicrobial molecules lose much of their activity, once attached to a surface. When providing an anchor for the active molecule through a flexible covalently bound polymeric chain, the active molecule should still be able to reach the site of action at or within the bacterium, e.g., by penetrating its cell wall, but leaching is still suppressed. Important parameters for polymer brush anchors are chain length and chain density. Polymer brushes have been shown to be effective for anchoring QACs or AMPs [36e40]. The idea behind is attractive unfortunately it is not feasible in a hospital environment. Again, we definitely don't employ QACs or AMP (antimicrobial peptides. MPs belong to te bodys defense mechanisms. However, three problems arise with AMPs.

a) AMPs have to be eluted from the surface and must be incorporated into the metabolism of microorganisms - activity by disruption of cell wall. Therefore, the activity is limited to a few days. (Own frustrating investigations over a period of 2 years!) Limited duration of activity

b) AMPs are not easy to be obtained. They could be obtained as Magainins from frog skin with very limited availability.

c) Synthesis of AMPs is not solved as AMPs are lethal factors for microorganisms.

Investigations over 2 years have been performed by AmiSTec. Synthetic AMPs induce fast resistance against microorganisms these microorganisms are in turn also insensitive to natural AMPs produced by the body. Definitely to be avoided! 
Using surface-initiated atom transfer radical polymerization, QACs with charge densities of $>1.5 \mathrm{e} 1015$ accessible quaternary amine units/cm2 were anchored through poly-2-(dimethylamino) ethyl methacrylate chains. Interestingly, these surfaces were bioactive even though the polymer chains were too short to penetrate the cells with envelope thicknesses of $46 \mathrm{~nm}$ for Gramnegative E. coli and $45.55 \mathrm{~nm}$ for Gram-positive Bacillus subtilis. The spectrum of activity has to be very broad. Surfaces are contaminated with a variety of microorganisms which affect each other limiting the spread and growth. If one species is eradicated the remaining microorganisms have the possibility to proliferate and spread uninhibited. Limited spectrum of activity is therefore entirely unacceptable. This demonstrates that surface charge density can be more important than chain length.

On the other hand, it was clearly shown that $\mathrm{N}$-alkyl-pyridinium exhibited high antimicrobial activity when anchored through a 750 or $25 \mathrm{kDa}$ poly-ethyleneimine (PEI) but showed no activity when using the $2 \mathrm{kDa}$ analogue. Therefore, only long-chained, moderately hydrophobic immobilized polycations exhibit microbicidal activity. Interestingly, polycationic polymer brushes are not subject to existing mechanisms of resistance such as multidrug resistance pumps or multidrug tolerance protein-expressing cells, presumably since there are no analogue structures in nature. Polycations on a surface are not heat resistant and cannot be extrusion molded, in addition the activity is obscured by any compounds e.g. grease, sweat etc. which are abundantly coating hospital surfaces close to patients. Anchoring AMPs is again not helpful, see above. In addition, the technology is complicated and expensive. These compounds cannot be added to various coatings.

Polymer brushes have also successfully been used for anchoring AMPs. AMPs are a logical alternative to conventional antibiotics due to their broad-spectrum antimicrobial activities. Surface concentrations of AMPs up to $5.9 \mathrm{mg} / \mathrm{cm}^{2}$ were achieved by conjugating the peptides to surface-immobilized primary amine functionalized polymer chains obtained by aqueous surface-initiated atom transfer radical polymerization of $\mathrm{N}, \mathrm{N}$ - dimethylacrylamide and aminopropyl methacrylamide hydrochloride. The efficacy of AMPs attached to catheter material surface using polymer brushes was verified in vivo by using a catheter-associated urinary tract infection mouse model.

By adding arginine glycine aspartate peptides to promote hosttissue cell adhesion to AMPs anchored through the block copolymer Pluronic F-127, two effects were achieved, namely thwarting bacteria from approaching and attaching to the surface and, simultaneously, enhancing tissue integration. A completely different approach is given by immobilizing bacteriophages on surfaces. Bacteriophages are viruses that infect bacteria and are highly efficient and relatively cost- effective. Bacteriophages are host specific, but they can have a broad host range, infecting several strains or species of bacteria, both Gram-positive and Gram-negative. They proved to be efficient in preventing bacterial contamination and recently they became accepted for food treatment to counter food contamination during storage. In addition, the fact that the EU is contributing V3.8 million to the Phagoburn study shows that it is willing to consider the approach.

Attachment of bacteriophages to a surface can be achieved through physisorption, electrostatic attachment, and covalent bonding. Sample surfaces, which exhibited antimicrobial activities with immobilized phages, included gold, glass, cellulose membrane, and hydrogels [44e47]. Phages are specifically sensitive to moisture and can be deactivated when dried. However, reactivation upon wetting is feasible, and addition of polysaccharides improves their stability. Phage therapy is probably inefficient for the prevention of hospital acquired infections in a hospital. It cannot be incorporated onto coatings on stainless steel, aluminum, enamel etc. as it is not heart stabile. Different microorganisms require different phages there is no uniform broad spectrum of activity against bacteria, fungi, molds and virus. Besides phages are not heat resistant and are also eluted from the surfaces with limited duration of activity. Two other groups of naturally occurring antimicrobials are claimed as alternatives to antibiotics: bacterial cell wall hydrolases (BCWHs) and antimicrobial peptides.

Antimicrobial peptides have a broad-spectrum against bacteria and fungus, low level of induced resistance, but may cause toxicity at high doses in order to be efficient and are more costly to produce. BCWHs have limitations towards Gram-negative bacteria, due to the presence of the outer membrane, and some important Grampositive pathogens such as S. aureus are already resistant to lysozymes.

\section{Biocide-Releasing Surfaces}

Biocide-releasing surfaces may have some conceptual disadvantages since they are toxic by design in terms of releasing biocidal substances. In addition, they will gradually become inactive and they may induce the formation of resistance. Any substance eluting from the surface is also emanating into the environment. Toxic substances may affect various cell lines in the body e.g. epithelia cells, osteoblasts, fibroblasts if incorporated into the body. The chance that these biocides meet the requirements of the European Commission is improbable and requires extensive testing. Catalytically active surfaces, such as photocatalytically active surfaces (e.g. $\mathrm{TiO}_{2}$ ) which regenerate reactive oxygen species upon UV radiation, provide an alternative. The activity of a photocatalytic mechanism e.g. oxygen radicals and hydroxyl radicals can only be documented with the JIS method. By this method the antimicrobial activity is determined in the capillary space between the surface and a foil which prevents these photocatalytic molecules from evaporating into the environment.

This is a highly arbitrary method of testing and is far away from the determination of an antimicrobial surfaces investigated by the 
push plate method (RODAC Plate method). One of the problems of a technology due to biocide releasing agents is the induction of resistance. There is a law by nature that all substances with antimicrobial activity which require the incorporation of the agent into the metabolism of microorganisms induce resistance. This is well known for antibiotics but also disinfectants. Therefore, it is required that a technology is developed which is not incorporated to the bacterial metabolism but attacks microorganisms from the outside i.e. acid water molecules, free radicals and most important a positive zeta potential i.e. a positive electrostatic charge at the surface which ruptures the phosphoplipid bilayer of microorganisms upon contact within minutes. Another approach is triggered release depending on certain threshold concentrations of quorum-sensing molecules which are found in biofilms.

a) Comment: The antimicrobial activity of a quorum sensing technology has never been documented beyond reasonable doubt. It is considered an interesting idea, but no one has ever been investigated the activity in comparison with other technologies. Surface coating with carbon nanotubes (CNTs), graphene or diamond-like carbons (DLCs) promised interesting antimicrobial activity, since these materials show relatively low cytotoxicity towards mammalian cells. Whether these materials are active on the surface or whether they achieve antimicrobial activity through releasing traces into the aqueous phase is not yet resolved, but their activity in microbial suspensions is clearly demonstrated, e.g. higher toxicity is found for surfactant-dispersed CNTs. The most frequently proposed mechanisms of action fall under four categories: (i) oxidative stress induction, (ii) protein dysfunction, (iii) membrane damage, and (iv) transcriptional arrest. Recently, it was also demonstrated that the mechanism of action depends on the concentration of the bacteriocide in this case graphene oxide (GO): low GO concentrations cut membranes of the microorganisms S. aureus and E. coli whereas high concentrations induce the formation of GO aggregates shielding their edges.

When cluster size increases, bacterial deactivation through wrapping is observed. Graphene-based materials differ in their morphology (mono and multilayers) as well as in their surface chemistry (graphene, GO, reduced graphene oxide (rGO)). Lateral size for instance is important to enhance bacterial adhesion whereas the sharp edges may act as nanoknifes. GOs can enhance the antimicrobial activity through oxidative stress with or without the production of reactive oxygen species. When comparing the antibacterial activity of graphite, graphite oxide, GO, and rGO towards E. coli under similar conditions, GO showed the highest antibacterial activity, followed by rGO, graphite, and graphite oxide. Synergistic effects are reported for graphene-based silver nanocomposites and composites with other antibacterial nanoparticles, as well as with polymeric or enzymatic bactericides. Graphene by itself show a very limited antimicrobial activity.

However, in combination with silver the antimicrobial activity is enhanced. However again only silver ions show antimicrobial activity and have to be incorporated into the metabolism of microorganisms: consequences: limited duration of activity whereas graphene is enhancing the release of silver ions. Carbon nanotubes have also been widely studied as antimicrobial material since they can be easily embedded into polymers. Again, a variety of morphologies has been studied such as single wall or multi-wall, but it seems that GO-based materials show higher antimicrobial activity.

b) Comment: Nanotechnology with the problems of meeting the requirements of the Biocidal product regulation, addition of silver for enhancement of antimicrobial activity. Synergistic effects were obtained by making composites of CNTs and chitosan within a hydrogel, or by decorating CNTs with poly(amidoamine)dendrimerimmobilized CDs and Ag2S quantum dots which enhanced the antimicrobial activity in solution. CNTs can also be used to prepare antimicrobial coatings either by electrodeposition of a polyvinyl-Ncarba-zoleeCNT film or by preparing spin-coated films. In the same work, the antimicrobial activity of dispersed CNTs was studied and it was found that such antimicrobial activity depended on the degree of dispersions. Antimicrobial activity of CNTs depends also on the length of CNTs, as was shown for poly (lactic-co-glycolic acid)-embedded CNTs, where the shorter ones were more active. Diamond-like carbons represent a further morphology of carbon materials. In contrast to graphite, graphene and CNTs, tetrahedrally structured amorphous carbons with $\mathrm{CeC}$ sp3 bonding are dominating with a significant amount of $\mathrm{CeH}$ bonds.

They can be prepared by chemical vapour deposition, e.g., on stainless steel surfaces, and they can be doped with known antimicrobial metals such as copper, silver, or platinum. When comparing the antimicrobial activity of pure DLCs and germaniumdoped DLCs, significant reduction in Pseudomonas aeruginosa biofilm formation was observed whereas these surface films showed no effect against Gram-positive S. aureus biofilms. Carbon quantum dots (CDs) are a relatively new class of carbon materials which can be used for bacterial identification due to their tunable photoluminescence properties. CDs exhibit low toxicity and appreciable biocompatibility. When decorating the surface of CDs with QACs or Ag NPs, it was possible to selectively attach C-dots to Gram-positive bacteria and to induce antimicrobial activity through the membrane-disrupting mechanism. Photocatalytic oxidation is a possible alternative strategy for antimicrobial coatings in the hospital environment.

Due to the self-regenerating biocidal effect of the catalytically released reactive oxygen species, such surfaces remain active throughout their lifetime. Many of the reported surfaces contain the photocatalyst $\mathrm{TiO} 2$, which generates highly active $\mathrm{OH}$-radicals in the presence of water, oxygen, and UV-A light. These highly reactive $\mathrm{OH}-$ radicals are able to destroy bacteria. Current research is focusing on shifting the photocatalytic activity of such coatings towards the visible light range, e.g. by adding silver nanoparticles which can act through their surface plasmon resonance effects, or molybdenum. When incorporating a combination of photosensitive dyes such as 
Crystal Violet with the inherently antimicrobial $\mathrm{ZnO}$ nano particles into polymer surfaces, synergistic photocatalytic antimicrobial activity was reported. The polymers exhibited significant bacterial kills using typical white light sources of hospital environments within $1 \mathrm{~h}$ against Gram-positive bacteria and within $6 \mathrm{~h}$ against Gram-negative bacteria.

By combining a dye with Ag nanoparticles, bactericidal activity of the Ag nanoparticles could be enhanced under white light illumination. It is believed that the enhancement effect is due to an increase in bactericidal activity through the triplet state of the dye by biomolecular reaction rather than by enhancement of the concentration of reactive oxygen species. Photocatalytic oxidation is a mechanism with a potential as it eradicates bacteria without incorporation into the bacterial metabolism. A further disadvantage is the requirement for an external light source. Prohibitive is that the technology can only be documented by the JIS method investigating the activity underneath a foil. Last not least free radicals don't penetrate into the thick layer of a biofilm. In situ generated surface by transition metal oxides surfaces decorated with metal oxide Lewis acids such as MoO3, WO3 and Zinc molybdate have also shown a broad-band and strong antimicrobial activity resulting in a reduction of the number of colony forming units by 6-7 log 10 within 1-3 hours.

Their mechanism of action is based on the in-situ generation of $\mathrm{H} 3 \mathrm{O}+$ ions through the reaction with moisture from the air inspired by the bodys own defense mechanism imitating e.g. the acid coating of the skin. The resulting acidified surfaces have a $\mathrm{pH}$ of 4.5 and the $\mathrm{H} 30+$ ions are able to diffuse through the cell membranes where they can distort the $\mathrm{pH}$-equilibrium and transport systems of the cell. In addition by this mechanism also free radicals e.g. oxygen radicals and hydroxyl radicals are formed which result in a synergistic mode of action including a positive zeta potential. This is reflected by an extraordinary fast eradication of microorganisms i.e. a reduction of $5 \log 10$ within 10 minutes.

c) Comment: This technology is the only one which meets all the requirements described initially for prevention of Hospital acquired infections. No induction of resistance, no allergenicity, the additives are essential trace elements in the body! Easy cleaning has been documented with water and detergents as microorganisms don't adhere on acid surfaces. The technology is also active against microorganisms embedded in a biofilm! Microorganisms in a biofilm are hibernating (it is a form of wintersleep) and don't take up anything from the outside. Therefore, all technologies which are based on incorporation of the antimicrobial agent into the bacterial metabolism are ineffective. Again, technologies which attack microorganisms from the outside also eradicate microorganisms in a biofilm an important asset. However also technologies based on oxygen radicals alone are also substantially less active as these free radicals are not able to penetrate the biofilm.

In addition also a positive Zeta potential is formed on the surface, responsible for the observed rapid eradication of microorgansism on surfaces documented by scanning electron microscopy. 6 log 10 reduction within 15 minutes. The technology is approved by the BPR as in situ generated biocide and is legitimately on the market. In the future no easy approval for in situ generated biocides is possible by ECHA, this opportunity expired September 1, 2018. This is also an additional favourable asset of the presently available technology. Reduced toxicity and prolonged durability of the antimicrobial effect may also be achieved by the triggered release of biocidal molecules. Recent strategies are based on quorum sensing: quorum-sensing molecules (e.g. homoserine lactones for Gram-negative bacteria) enable bacteria to detect the presence of other bacteria and to communicate with them. The concentration of quorum-sensing molecules increases with bacterial multiplication and at certain threshold concentrations the expression of many genes is affected, such as genes encoding for adhesion or lipases, which are particularly abundant at sites of infection.

d) Comment: The antimicrobial activity of a quorum sensing technology has never been documented beyond reasonable doubt. It is considered an interesting idea but no one has ever been investigated the activity in comparison with other technologies. The "idea" is in the air since 20 years no effective samples have ever been presented with documented antimicrobial activity. The technology also requires approval of the BPR of the European Union. By coupling the antibiotic ciprofloxacin through a lipase-sensitive homoserine group on to the surface of a PEG model compound, a selfregulating system was obtained. Alternatively, antiquorum sensing enzymes could prevent bacteria from forming biofilms by suppressing the quorum-sensing molecule concentration below the threshold value. The incorporation of antibiotics in particular results in the induction of resistance to a group of antibiotics indispensable in clinical practice. The duration of activity is limited to a few days.

This is in no way a practical approach and has to be banned. Also the incorporation of disinfectants has to be banned due to the induction of cross resistance with antibiotics,Many plant extracts are well known for their antimicrobial properties and much research is devoted to their application to protect food from pathogens. However, limited research has been done on investigating their efficacy on surfaces of healthcare units or on medical devices, such as tympanostomy tubes. It has been shown that a tea-tree oil coating may induce zones of inhibition against MRSA after a twoday incubation. Plant extracts may exhibit antimicrobial activity by fermentative properties and formation of free fatty acids. There are other less well exactly documented mechanisms. Plant extract however are not heat resistant, difficult to work with and water soluble. No license by the BPR.

\section{Impact of Topography on Surface Effectiveness}

It is generally acknowledged that defects or design features on any inert surface can retain soil and/or micro-organisms, and therefore affect cleanability, disinfection, and hygienic status of the surface. Implications in the clinical environment in terms of cross- 
infection control, the choice of surface material to be used, and the cleaning and sanitization protocols are significant. However, the assumption 'the rougher the surface, the worse the hygienic status' is somewhat simplistic, although many publications make this type of claim. Cells are easily removed from 'smooth' surfaces, but they may be retained within features approximating in size to that of the cells. In larger features, the cells may again be relatively easily removed. Easy cleanability is in addition to an optical attractive surface one of the prerequisites. If easy cleaning is also accompanied by a germfree surface this is the ideal approach as this can avoid the use of disinfectants which hence are responsible for the dramatic induction of resistant microorganisms seen.

Technologies which combine easy cleaning and antimicrobial activity are preferable. It has been documented that the technology with surfaces decorated with metal oxide Lewis acids such as MoO3, W03 and Zinc molybdate have also shown a broad-band and strong antimicrobial activity resulting in a reduction of the number of colony forming units by $6-7 \log 10$ within 3 hours. Their mechanism of action is based on the in-situ generation of $\mathrm{H} 3 \mathrm{O}+$ ions through the reaction with moisture from the air inspired by the bodys own defense mechanism e.g. the acid coating of the skin. The resulting acidified surfaces have a pH of 4.5 and the $\mathrm{H} 30+$ ions are able to diffuse through the cell membranes where they can distort the pH-equilibrium and transport systems of the cell. In addition by this mechanism also free radicals e.g. oxygen radicals and hydroxyl radicals are formed which result in a synergistic mode of action in cluding a positive zeta potential. This is reflected by an extraordinary fast eradication of microorganisms i.e. a reduction of $5 \log 10$ within 10 minutes.

Cleaning 2 hours after deposition of 109 colony forming units per $3 \mathrm{~cm}^{2}$ with a paper towel immerse in water demonstrated complete eradication of microorganisms. Self-disinfecting surfaces are highly cost efficient as the save the daily application of disinfectants for years. At the same time the emergence of resistant microorganisms is prevented by stopping the application of disinfectants. Typically, surface topography is measured by the Ra-value, defined as 'the average departure of the surface profile from a center line'. Other parameters are also used, but the Ra-value is the most popular in the microbiology literature. An Ra-value of $0.8 \mathrm{~mm}$ is often deemed indicative of a hygienic surface. In profilometry, a trace is taken of the surface, typically perpendicular to the lay of the surface features, using a probe. However, the probe will itself vary in size, depending on the method used to assess topography, from solid stylus, through laser scanning to the nanoscale tip of the atomic force microscope (which generates Ra-values in nanometers).

The resolution of these different probes will affect the result obtained, although, using standardized surfaces of different degrees of roughness, the ranking (if not the absolute measurement) would likely bethe same irrespective of the method used. In addition, the profilometer might reveal a two- or three-dimensional impression of the surface. If two-dimensional, then the overall picture of the surface is not revealed: a feature identified along a linear trace might indicate a scratch or a pit e the type as well as degree of roughness may be important in terms of microbial retention. Threedimensional images are more valuable, for example revealing very different topographies for surfaces with comparable Ra-values. Since the Ra-value is a statistical measure, then the actual feature size, or the variation in feature size across a surface, is not revealed. This may be important if features of the dimension of microbial cells are present within larger features, with macro-, micro- and nano features each potentially having different effects on the retention of cells on the surface. Indeed, the previously mentioned 'lotus effect' reveals that a hierarchical micro/nanostructure can significantly reduce retention, enabling cells to 'roll off' the surface.

The fabrication of surfaces with well-defined nano-topographies provides a new avenue for the design of anti-adhesive/easily cleanable (and therefore hygienic) surfaces e depending on the intended environment of use. The Lotus effect has been investigated in great detail a number of years ago; however, the efficacy was very limited. It has been documented that bacteria adhere less vigorously on a microrough surface. This requires however an active elimination of microorganisms by a constant flow of water or constant active wiping of the surface. The environment in which surfaces are placed will also affect their hygienic status. At a flowing solid- liquid interface, cells will move across the surface, and may be retained in features where they may replicate and form biofilms with accompanying 'streamers' which may detach and contaminate downstream. This is a particular issue with joints in pipework. However, on open surfaces, at a solid air interface, the cells tend to be deposited on the surface through contact with vectors such as food, fingers, equipment, or splashing.

In this case, replication is less likely, since water availability is low, and the survival of cells on these surfaces is key to maintaining hygienic status. Antimicrobial surfaces, and/or surfaces which are hard or difficult to abrade, coupled with effective cleaning regimes, are strategies employed to counter this phenomenon. The continued cleaning/soiling cycle can itself affect the surface, causing abrasions that result in increased soiling and require increasing force in cleaning which in turn may increase abrasion. The nature of the surface itself can affect how it wears: steel and other metals tend to scratch; glass and ceramics tend to fracture; softer materials such as plastics will abrade more easily. Antimicrobial surfaces that actively leach out active agents might prove more effective if the surface area is increased through abrasion, but the presence of retained organic material (blood, food, sputum) in addition to micro-organisms might impede the antimicrobial effect and protect the microbial cells. It might be argued that the increase in surface area presented by surfaces with increased roughness is the driver for the increased retention, but this has not been convincingly proven.

The features themselves, in terms of shape, profile, and size clearly provide an increased area of contact for cells, enhancing 
their ability to remain on surfaces. All of these issues should be considered when developing novel and effective antimicrobial surfaces, focusing on minimizing wear to maintain cleanliness and cleanability. At the cellular level, several studies focus on the retention of cells on surfaces. The typical experiment involves the incubation of surface with cell suspension for a specified timeperiod (such as $1 \mathrm{~h}$ ), rinsing and removal prior to staining retained cells, and quantifying the amount of retention (cell numbers per unit area, or area of microscopic field covered by cells). However, one might debate which is more desirable: high numbers of retained cells which are easily removed, in comparison to low numbers of retained cells which resist detachment. Here, issues of cell survival and inactivation are also important. The atomic force microscope is one means of assessing the strength of attachment of cells on a surface.

The probe scans repeatedly across the surface, moving vertically in response to surface features. This movement is captured and imaged using lasers. By increasing the force of the scan, less strongly attached cells are removed. Thus, the strength of attachment as well as the amount of retention can be assessed. This work has revealed that the size of cells and their relationship with the feature size affect strength of attachment: as might be expected, comparable feature size and cell size is the least desirable combination, enabling maximum contact area between cell and surface. In addition, cell shape will also affect this interaction, with rod-shaped cells having a larger area of contact available for interaction with the cell surface in comparison to cocci. Investigation of the strength of attachment of cells on linear features where the force is applied either across or along the feature has revealed different results: demonstrating easier removal along well-defined features on titanium-coated stainless steel, but easier removal by applying force across features on softer polymeric surfaces.

As noted above, this work has led to the fabrication of surfaces with designed topographies that are targeted at inhibiting attachment of particular cells, where surface features smaller than cells might reduce their ability to strongly attach to the surface, and therefore improve cleanability. The robustness of these surfaces is essential to ensuring a long- lasting effect, and the potentially interfering effect of organic material must also be considered. When considering open surfaces that are usually present at a solideair interface, which is the main focus of this paper, biofilms are of less concern. In the clinical/medical environment, hightouch surfaces (worktops, walls, doorhandles, telephones, patient surrounds) are the prime focus for antimicrobial treatments and/ or effective cleaning. Solide-liquid interfaces, where biofilms could form, would likely be encountered around taps, showers or drains. The topography of the surface underlying the biofilm does not necessarily influence the quantity of the biofilm itself again depending on the scale of feature size e but after cleaning, the substratum will retain cells in features which can regrow and reduce the time taken for the biofilm to develop once more.
Microorganisms in a biofilm cannot be eradicated by antibiotics and disinfectants. The reason: Bacteria in a biofilm are hibernating i.e. the don't take up anything (biocides, antibiotics etc.) from the environment. It is of crucial importance that selfsanitizing surfaces prevent the formation of a biofilm or are active against microorganisms in biofilms! The effects of photoactivated $\mathrm{TiO}(2)$ on microorganisms has been evaluated. The activity has been shown to be capable of killing a wide range of Gram-negative and Grampositive bacteria, filamentous and unicellular fungi, algae, protozoa, mammalian viruses and bacteriophage. Resting stages, particularly bacterial endospores, fungal spores and protozoan cysts, are generally more resistant than the vegetative forms, possibly due to the increased cell wall thickness. The killing mechanism involves degradation of the cell wall and cytoplasmic membrane due to the production of reactive oxygen species such as oxygen radicals, hydroxyl radicals and hydrogen peroxide.

This initially leads to leakage of cellular contents then cell lysis and may be followed by complete mineralization of the organism. Killing is most efficient when there is close contact between the organisms and the $\mathrm{TiO}(2)$ catalyst. The documentation of the antimicrobial activity proved to be a problem. Oxygen radicals and hydrogen peroxide evaporates rapidly from the surface and the time in contact with pathogens is not sufficient. Therefore, a new method (Japanese industrial standard JIS 25923) has been designed where the surface is covered with a foil after inoculation with germs. This prevents free radicals from evaporate into the environment. This method is an arbitrary test system far away from the real-life situation and has major disadvantages. First: in the capillary space between the surface and the foil an unrealistic high concentration of free radicals accumulates which is responsible for the documentation of an antimicrobial activity which is not present in the real life situation. Second: there is no interest in the investigation of an antimicrobial surface underneath a foil. A realistic test system e.g. the push plate method does not disclose the an antimicrobial activity of this technology.

However also a number of other more potent catalysts have been investigated with superior antimicrobial activity. In situ generated biocides on a surface by incorporation of transition metal oxides e.g. Lewis acids such as MoO3, WO3, Zinc molybdate and polyoxometallates show an excellent antimicrobial activity and meet all the necessary requirements for prevention of hospital acquired infection and to halt the emergence of multiresistant microorganisms described above. Surfaces decorated with the transition metal oxide molybdenum oxide have also shown a broadband and strong antimicrobial activity resulting in a reduction of the number of colony forming units by $6-7 \log 10$ within 1-3 hours. Their mechanism of action is based on the in-situ generation of $\mathrm{H} 3 \mathrm{O}+$ ions through the reaction with moisture from the air inspired by the bodys own defense mechanism imitating e.g. the acid coating of the skin. 
The resulting acidified surfaces have a $\mathrm{pH}$ of 4.5 and the $\mathrm{H} 3 \mathrm{O}+$ ions are able to diffuse through the cell membranes where they can distort the $\mathrm{pH}$-equilibrium and transport systems of the cell. In addition also free radicals e.g. oxygen radicals and hydroxyl radicals are formed which result in a synergistic mode of action and can be documented by the push plate (RODAC plate) method. Last but most important a positive zeta potential is formed with a positive electric charge in a distance of a few micrometers at the surface. This electric charge attracts electronegative charged microorganism. Upon contact the phospholipid membrane is disrupted within minutes a documented by laser scanning microscopy. This is reflected by an extraordinary fast eradication of microorganisms i.e. a reduction of $5 \log 10$ within 10 minutes. The antimicrobial activity is very broad and includes Gram positive and Gram negative microorganisms regardless of the resistance to antibiotics, fungi many virus e.g. influenza, Epstein Barr virus, Herpes viurus jus to name a few.

This technology is the only one which meets all the requirements described initially for prevention of Hospital acquired infections. No induction of resistance is seen. A tolerance study with 10 persons who had a $8 \times 8 \mathrm{~cm}$ plate endowed with Zinc Molybdate $2 \%$ in melamine resin on their forearm in a wet chamber disclosed no side effects within an observation time of 120 hours. No side effects have been observed as the surface $\mathrm{pH}$ of the samples corresponded to the normal acidity of the skin. Investigations of elution of the additive has been investigated The additives are essential trace elements in the body! No allergenicity has been detected within the next 2 years of observation. Cleaning has been investigated: Gloss paint samples and Zinc Molybdate 2\% melamine resin coated boards have been cleaned with water and detergents. No loss of antimicrobial activity is observed after 10000 cleanings. Neither zinc nor molybdenum has been detected by spectro turboquant powder in thje textile.

The technology is also active against microorganisms embedded in a biofilm where antibiotics and disinfectants are ineffective! Microorganisms in a biofilm are hibernating (it is a form of wintersleep) and don't take up anything from the outside. Therefore, all technologies which are based on incorporation of the antimicrobial agent into the bacterial metabolism are ineffective. Again, technologies which attack microorganisms from the outside also eradicate microorganisms in a biofilm an important asset. However also technologies based on oxygen radicals alone are also substantially less active as these free radicals are not able to penetrate the biofilm. In addition, also a positive Zeta potential is formed on the surface, responsible for the observed rapid eradication of microorganisms on surfaces documented by scanning electron microscopy. $6 \log 10$ reduction within 15 minutes has been observed. The technology is approved by the BPR as in situ generated biocide and is legitimately on the market.

In the future no easy approval for in situ generated biocides is possible by ECHA, this opportunity expired two years ago. This is also an additional favorable asset of the presently available technology. Surveillance and Response. Situational awareness is crucial for addressing national and international threats. Yet, the world currently lacks comprehensive monitoring for antibiotic resistance emerging domestically and being imported from abroad. The surveillance systems are woefully underfunded. Powerful tools that emerged from the Human Genome Project have the ability to reveal how antibiotic resistance arises and spreads across health care facilities, agriculture, the environment and international borders, but they are not being routinely used. And adequate public and animal health infrastructure for monitoring antibiotic use and resistance is lacking in many states and localities. Specific actions and investments intended to achieve better surveillance, stewardship of currently used antibiotics, and development of new antibiotics.

\section{References}

1. Magill SS, Edwards JR, Bamberg W, Lucy E Wilson, Scott K Fridkin, et al. (2014) Multistate Point-Prevalence Survey of Health Care-Associated Infections. N Engl J Med 370(13): 1198-1208.

2. Protano C, Cammalleri V, Romano Spica V, Valeriani F, Vitali M (2009) Hospital environment as a reservoir for cross transmission: cleaning and disinfection procedures Ann Ig. Review 31(5): 436-448.

3. Langsrud S, Sidhu MS, Heir E, Holck A (2003) Bacterial disinfectant resistance- a challenge for the food industry. Int Biodeter Biodegr 51(4): 283-290.

4. (2009) The world health organization: WHO Guidelines on Hand Hygiene in Health CareFirst Global Patient Safety Challenge Clean Care is Safer Care. Geneva: World Health Organization.

5. Machowska A, Stålsby Lundborg C (2018) Drivers of Irrational Use of Antibiotics in Europe. Int J Environ Res Public Health 16(1): 27

6. Weiss RA, McMichael AJ (2004) Social and environmental risk factors in the emergence of infectious diseases. Nat Med 10: S70-S76.

7. Begrow F, Böckenholt C, Ehmen M, Wittig T, Verspohl EJ (2012) Effect of myrtol standardized and other substances on the respiratory tract: ciliary beat frequency and mucociliary clearance as parameters. Adv Ther 29(4): 350-358.

8. Kastner U, Glasl S, Follrich B, Guggenbichler JP, Jurenitsch J (2002) Acid oligosaccharides as the active principle of aqueous carrot extracts for prevention and therapy of gastrointestinal infections. Wien Med Wochenschr 152(15-16): 379-381.

9. Guggenbichler JP (1982) [Studies on resorption of orally administered antibiotics and chemotherapeutic agents in children and its modification. 2] Pädiatr Padol 17(3): 565-584.

10. Kastner U, JP Guggenbichler JP (2001) Influence of macrolide antibiotics on promotion of resistance in the oral flora in children Infection 29(5): 251-256.

11. Møretrø T, Langsrud S (2017) Tolerance to quaternary ammonium compound disinfectants may enhance growth of Listeria monocytogenes in the food industry and induced cross resistance with antibiotics. Int J Food Microbiol 241: 215-224.

12. Hall CW, Mah TF (2017) Molecular mechanisms of biofilm-based antibiotic resistance and tolerance in pathogenic bacteria. FEMS Microbiol Rev 41(3): 276-301.

13. Dizaj SM, Lotfipour F, Barzegar Jalali M, Zarrintan MH, Adibkia K (2014) Antimicrobial activity of the metals and metal oxide nanoparticles. Mater Sci Eng C Mater Biol Appl 44: 278-84. 
14. Guggenbichler JP, Assadian O, Boeswald M, Kramer A (2011) Incidence and clinical implication of nosocomial infections associated with implantable biomaterials - catheters, ventilator-associated pneumonia, urinary tract infections Krankenhhyg Interdiszip 6(1).

15. Guggenbichler JP (2002) Intensive own investigations.

16. Walczak M, Richert A, Burkowska But A (2014) The effect of polyhexamethylene guanidine hydrochloride (PHMG) derivatives introduced into polylactide (PLA) on the activity of bacterial enzymes. J Ind Microbiol Biotechnol 41: 1719-1724.

17. Oh KB, Lee JH, Lee JW, Yoon KM, Chung SC, et al. (2009) Synthesis and antimicrobial activities of halogenated bis(hydroxyphenyl)methanes. Bioorg Med Chem Lett 19(3): 945-948.

18. Soliman GM, Attia MA, Mohamed RA (2014) Poly (ethylene glycol)block-poly( $\varepsilon$-caprolactone) nanomicelles for the solubilization and enhancement of antifungal activity of sertaconazole. Curr Drug Deliv 11(6): 753-762.

19. Liu JY, Sonshine DA, Shervani S, Hurt RH (2010) Controlled Release of Biologically Active Silver from Nanosilver Surfaces. Acs Nano 4(11): 6903-6913.

20. Gomes AP, Mano JF, Queiroz JA, Gouveia IC (2015) Incorporation of antimicrobial peptideson functionalized cotton gauzes for medical applications. Carbohydr Polym 127: 451-461.

\section{ISSN: 2574-1241}

DOI: 10.26717/BJSTR.2021.34.005491

J Peter Guggenbichler. Biomed J Sci \& Tech Res

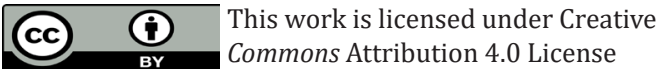

Submission Link: https://biomedres.us/submit-manuscript.php
21. Pedrosa M, Mouro C, Nogueira F, Vaz J, Gouveia I (2014) Comparison of the antibacterial activity of modified-cotton with magainin I and LL-37 with potential as wound-dressings. Journal of Applied Polymer Science.

22. Gilbert P, Moore LEN (2005) Cationic antiseptics: diversity of action under a common epithet. J Appl Microbiol 99(4): 703-715.

23. Hasan J, Crawford RJ, Ivanova EP (2013) Antibacterial surfaces: the quest for a new generation of biomaterials. Trends Biotechnol 31(5): 295-304.

24. Zhang X, Wang L, Levänen E (2013) Superhydrophobic surfaces for the reduction of bacterial adhesion. RSC Advances.

25. Zhu H, Guo Z, Liu W (2014) Adhesion behaviors on superhydrophobic surfaces. Chem Commun (Camb) 50: 3900-3913.

26. Yan YY, Gao N, Barthlott W (2011) Mimicking natural superhydrophobic surfaces and grasping the wetting process: a review on recent progress in preparing superhydrophobic surfaces. Adv Colloid Interface Sci 169(8): 80-105.

27. Feng L, Li SH, Li YS, Li HJ, Zhang LJ, et al. (2002) Super-hydrophobic surfaces: From natural to artificial. Advanced Materials 14(24): 18571860.

28. Yang WJ, Cai T, Neoh KG, Kang ET, Teo SL, et al. (2013) Barnacle cement as surface anchor for "clicking" of antifouling and antimicrobial polymer brushes on stainless steel. Biomacromolecules 14(6): 2041-2051.

$\begin{array}{ll}\text { BIOMEDICAL } & \text { Assets of Publishing with us } \\ \text { RESEARCHES } & \text { - Global archiving of articles } \\ \text { - Immediate, unrestricted online access }\end{array}$

